\title{
A Proposal of Sensor Data Collection System Using Mobile Relay Nodes*
}

\author{
Ryota Ayaki, Hideki Shimada, Kenya Sato \\ Department of Information Systems Design, Doshisha University, Kyoto, Japan \\ Email: ayakix1935@gmail.com, hideki-s@is.naist.jp, ksato@mail.doshisha.ac.jp
}

Received October 16, 2011; revised November 23, 2011; accepted December 20, 2011

\begin{abstract}
In recent years, as embedded devices become smaller, cheaper and more diverse, the demand for urban sensing systems that present valuable information to users is increasing. However, in achieving urban sensing systems, the communication channel from the sensors to the data centers pose a problem, especially in respect to the cost of furnishing IP/mobile networks for each and every one of the sensor nodes. Many existing researches attempt to tackle this problem, but they generally limit either the types of sensors used or the distances among the sensors. In this paper, we propose a new sensor data collection system model in which mobile relay nodes transport the sensor data to the data center. We ran simulations under conditions imitating the real world to verify the practicality of the proposed system. This simulation uses data accumulated from traffic surveys to closely imitate pedestrians in the real world. We evaluated that the proposed system has sufficient ability to use in urban sensing systems that are not under the real-time constraint.
\end{abstract}

Keywords: Wireless Sensor Networks; Data Aggregation; DTN; Mobile Networks

\section{Background}

In recent years, as sensors become smaller, cheaper and more diverse, a wide range of matter is becoming subject to sensing. For instance, this includes natural phenomena such as earthquakes and weather, words said by people, train schedule delays, social phenomena such as sales and opening hours at shops, and man-made objects such as products and buildings. As more and more things become subject to sensing, more information such as what is happening where, who is doing what can be used in our everyday environments. Urban sensing systems that collect a variety of data using a multitude of sensors and provide them to users as beneficial information are gaining demand.

A variety of sensing models are proposed to achieve urban sensing systems. An example would be one using ad-hoc networks where sensor nodes attached to the environment send their data wirelessly to other nodes without using a base station $[1,2]$. In this paper we will call this the "fixed-sensor" sensing model. Another example would be one where humans or robots equipped with sensors use store-and-forward networking to collect data as they roam around the environment $[3,4]$. In this paper we will call this the "roaming-sensor" sensing model.

There are many applications of urban sensing. In particular, video monitoring systems for security surveillance

*This work was supported by JSPS Grant-in-Aid for Scientific Research KAKENHI (2100084). and elder-care have high demand [5,6]. These systems require that footage be collected from numerous cameras placed around the area. However, the fixed-sensor model, if it were to be employed in this system, requires other nodes to be present inside the wireless networking area at all times. The roaming-sensor model, on the other hand, is difficult to use in situations where sensors need to be fixed, and it also limits the types of sensors that can be used.

This paper proposes a new sensing model, the "mobile relay node" sensing model, where nodes that transmit data are physically detached from nodes that collect data. More specifically, relay nodes that roam around the area receive data from fixed sensors and transmit them to data centers. To discuss the practicality of this system, we ran simulations imitating the real world and evaluated the data collection ability.

\section{Related Studies}

\subsection{Fixed-Sensor Sensing Model}

Wireless sensor networks use wireless networking among sensor nodes to collect data from a number of distributed sensors. The most popular network model used is the ad-hoc network where nodes communicate directly with each other without routing through data centers. This is an effective way of building a low-cost network where infrastructure is not sufficient. However, because ad-hoc networks use multi-hop communication, it requires that nodes 
have other nodes present within their wireless areas at all times. For this reason, although ad-hoc networks are effective in settings where sensors are placed in a limited area, they are unsuitable for settings where sensors are distributed over a wider area (such as in urban sensing).

One application of the wireless sensor network is the Field Server [7], an animal and plant growth monitoring system.

\subsection{Roaming-Sensor Sensing}

DTN (Delay/Disruption-Tolerant Network) [8] is a network architecture that achieves high-reliability data transmission in environments where disruptions, disconnections and major transmission delays occur frequently. DTNs use relay nodes that hold on to the data while moving around and transmit them when another node comes into its communicable area. This is called store-and-forward.

One application of the DTN is DakNet [9]. DakNet's main components are the Internet access point, the mobile access point, and the kiosks. The Internet access point has access to the Internet. The mobile access point is the relay node, usually a motor vehicle that runs a certain route periodically, such as a bus or motorcycle. The kiosks are end user terminals. DakNet allows the users to (indirectly) connect to the Internet through their kiosks. However, as we mentioned above, DakNet uses buses or motorcycles as relay nodes and they only go through a certain route, and is difficult to apply to urban sensing where the target area is relatively wide.

Human Probe [10] is a sensing model where humans equipped with wearable sensors roam around the target area (mainly urban districts). The persons carrying the sensors are to also carry a networking device so that they can transmit the data to data centers. However, because wearable sensors are limited by their weight, size and shape, many types of data are uncollectable.

Car Probe [11,12] uses cars as moving sensors. This system is categorized in ITS (Intelligent Transport Systems), and there are various applications [13]. In Car Probe, sensor data is collected as the car runs, then coupled with GPS data and accumulated in the data center. Data in the data center can then be used to form information on traffic congestion, traffic accidents and weather, and these can in turn be provided to drivers. For example, windshield wiper operation data can provide rain information, and ABS operation data can provide information on frozen road surfaces. However, Car Probe is unsuitable for situations where sensors need to be in a fixed position.

\section{Proposed System}

\subsection{Overview}

In this paper, we propose a new sensor data collection model, the "mobile relay node" sensing model, where nodes that transmit data are physically detached from nodes that collect data. More specifically, relay nodes that roam around the area receive data from fixed sensors and transmit them to data centers.

In contrast to fixed-sensor sensing, the mobile relay node model does not require that sensor nodes always have other nodes within their communicable area. In contrast to roaming-sensor sensing, the mobile relay node model is not limited in its range area or usable sensor types.

\subsection{Assumed Environment}

The proposed system is composed of the sensor nodes, the mobile relay nodes, and the data center.

The sensor nodes are sensors eccentrically placed in the environment that collect data on natural or social phenomena or man-made objects. Some examples are luminometers, thermometers and cameras. Sensor nodes in urban sensing systems need to be particularly large in number and, at the same time, inexpensive to produce and maintain. Therefore, we assume that they do not have the ability to connect to mobile wide-area data networks. These sensor nodes use wireless PAN (Personal Area Networking) to communicate with the mobile relay nodes.

The mobile relay nodes are moving objects with no fixed routes. That is to say, the nodes do not have a certain route that they must follow, and has no or few limitations on where they can go. Humans, animals or motor vehicles are suitable for these nodes. The mobile relay nodes are to have both wireless PAN and mobile widearea data network connectivity. As they roam around the environment, the mobile relay nodes collect data from sensor nodes using wireless PAN and are responsible for relaying the data to the data center.

The data center is a base station or server connected to the Internet and is used to accumulate the data collected by sensor nodes. It is to have a database for storing the data and is to perform computations on the data and possibly publish the resulting information.

\subsection{Sensor Data Collection Model}

Figure 1 shows the sensor data collection model. The curved lines indicate the routes that mobile relay nodes pass through. The dotted lines indicate data collection and transmission

The list below is the flow of operations.

1) The mobile relay nodes set their wireless PAN to receiving mode.

2) The sensor nodes sense the environmental data and store them in their queue buffer.

3) Once data is stocked in their buffers, the sensor nodes use wireless PAN such as UWB (Ultra Wide Band), Zig- 
Bee, or Bluetooth to search a nearby mobile relay node.

4) When a mobile relay node comes in a sensor node's wireless PAN area and receives the search signal, it issues a connection permission and data transmission command.

5) The sensor node, upon receiving these signals, uses the wireless PAN to send the data stocked in its buffer to the mobile relay node.

6) The mobile relay node uses its wide-area data network connection to forward the data to the data center.

\subsection{Assumed Applications}

Because the proposed model uses sensor nodes and mobile relay nodes that are physically detached from each other, implementers can choose the best type of mobile relay node to use depending on the setting. For instance, in urban districts with many pedestrians, mobile phones can be used as mobile relay nodes. This setup would provide a large number of mobile relay nodes and would be able to collect a great amount of sensor data.

Although the mobile relay node model does not yield real-time performance, the flexible characteristic pointed out above allows a wide variety of applications. One example is a wide-range video surveillance system. Currently, surveillance cameras are placed in crowded areas such as convenience stores and train stations. The cameras' primary purpose is security, and the number of cameras is not large. Also, the video data is generally only provided to the owners of the cameras and not published. On the other hand, a system that uses the proposed model allows a large number of video cameras to be placed throughout the city. The video data accumulated can possibly be used to provide end-users with information on what is happening where, and who is doing what.

\section{Simulated Environment}

\subsection{Overview}

We have built a simulator in order to evaluate the proposed model's ability to collect sensor data. Because sensor data generally lose value as time passes in urban sensing systems, we evaluate the model's ability to collect data based on latency, as in the elapsed time since sensing up to collection by the mobile relay node.

The simulated environment is based on the assumed application stated in Section 3.4. The sensor nodes are video cameras and the mobile relay nodes are handheld devices carried around by humans. The video data acquired by the video cameras are to be ultimately sent to the data center by the handheld devices.

\subsection{Initial Data}

In order to run the simulation, we needed a set of initial data regarding how the humans, or mobile relay nodes, move around.
The People Flow Project [14], conducted by The Center for Spatial Information Science at The University of Tokyo, provides "People Flow" data. This is a set of computed data collected from traffic surveys in metropolitan districts. The traffic surveys investigate many aspects of human traffic, including the types of people, their objectives, their origins and destinations, and the modes of transportation. The surveys target approximately $2 \%$ of the total population.

Figure 2 shows a sample of the traffic survey data. The surveys handle "trips" as basic units of human flow: a trip starts when a person starts travelling from a certain origin and ends when she arrives at a certain destination. The figure shows three trips, one starting at home and ending at the office (the commute), another starting at the office and ending at the shop (the shopping), and a third starting at the office and ending at home (the return). Although the traffic surveys provide data on the modes of transportation and the starting and ending timestamps of the trips, the raw data does not contain location data (as in latitude and longitude). The People Flow Project is helpful here as it performs computations on the original survey data to infer location data on the humans, and the resulting data is available through its Web API.

In our simulation, we used this Web API to extract pedestrian data and used it as initial data for pedestrian routes.

\subsection{Building the Simulator}

We have built a simulator using Java. As mentioned in Section 4.2, routing of the mobile relay nodes are based on pedestrian trip data extracted from People Flow data. The trip data are acquired from a sample number approximating to $2 \%$ of the total population. Although nearly 50 times this number is assumed to be present in the actual environment, we have not altered the number of pedestrians because it is difficult to accurately infer their routes. This means that in the simulation, the $2 \%$ of the population who participated in the traffic survey carry handheld devices that act as mobile relay nodes.

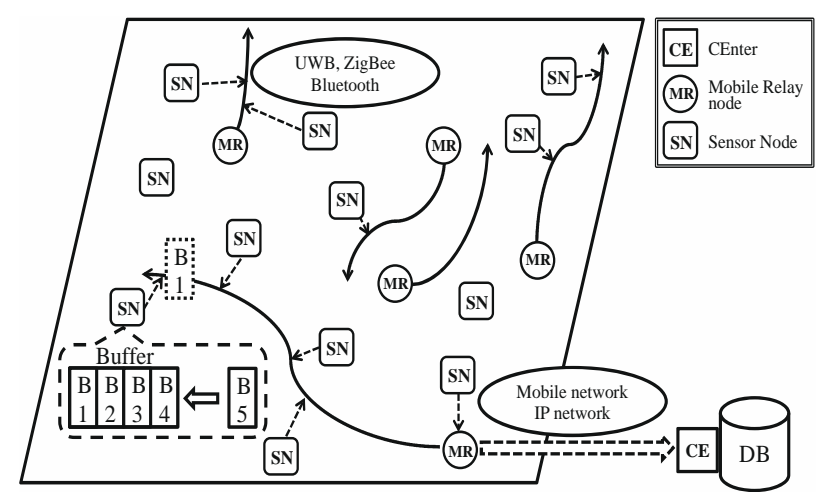

Figure 1. Model of sensor data acquisition. 


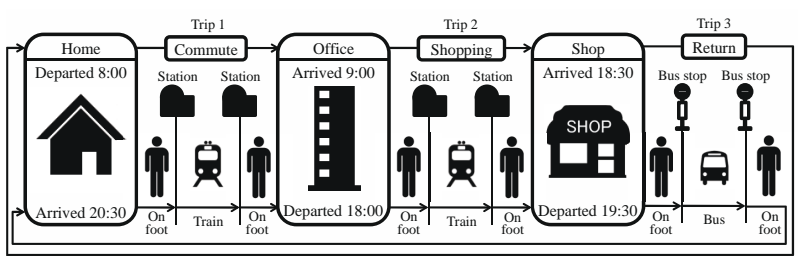

Figure 2. A sample of the traffic survey data.

\section{Evaluation and Discussions}

\subsection{Picking Out the Initial Data}

The list below is the flow of operations.

Requirement 1) Transit infrastructure such as roads and railroads are sufficient.

Requirement 2) The area is stably crowded.

Requirement 3) A portion of the area is off-limits to motor vehicles.

Requirement 1 is to ensure that pedestrian routes are relatively easy to infer from trip data. Requirement 2 is to ensure that the data is not biased due to insufficient number of samples. Requirement 3 ensures that the characteristic discussed in Section 3.4 is visible in the simulation. That is to say, the implementer can choose her optimal type of mobile relay nodes depending on the amount of sensor data and the placement of the sensors, and handheld devices that cannot be employed in DakNet or Probe Car are suitable for mobile relay nodes.

Based on these requirements, we chose a $5 \mathrm{~km}$ square area centered on Tokyo Metropolitan City Hall.

Figure 3 shows a day's worth of pedestrian trip data by time. This shows that there is a rise at around 7:00 and a gradual fall starting at around 18:00. Therefore we used the data from 8:00 through 19:00 where the number of trips is relatively stable. The total number of trips within this time slot is 331,303 , and the average hourly number of trips is 30,118 . The number of unique persons that passed through this area in this time slot is 18,342 , and the hourly average is 1667 . The reason why Figure 3 shows spikes at the tops and bottoms of the hours is because the traffic surveys are performed as questionnaires at those certain points of time.

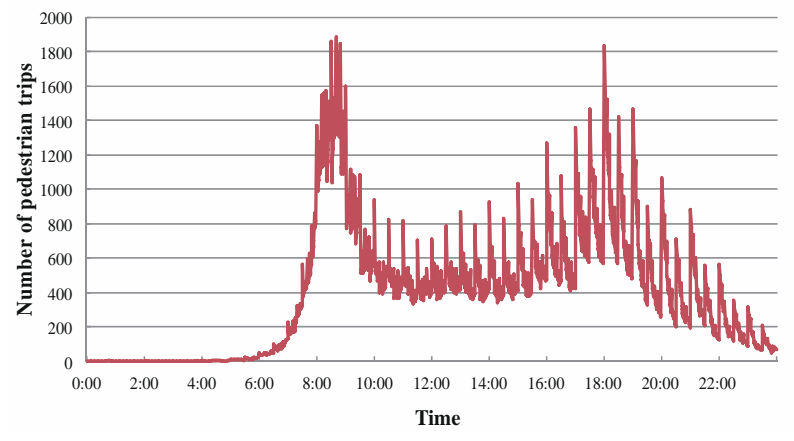

Figure 3. Transition of pedestrian trips.

\subsection{Picking Out the Initial Data}

Table 1 shows the parameters used in the simulation. Data generations at the sensor nodes are to take place every 1 minute. Because we ran the simulation at a 1-minute resolution, this means that data is accumulated continuously. Data sizes at the sensor nodes are 100, 300, 500, 700 and 900 KBytes. Communicable proximity between a sensor node and a mobile relay node using PAN is to be $10 \mathrm{~m}$. Data transmission rate using PAN is to be $1600 \mathrm{Kbps}$. These numbers are based on Bluetooth's capabilities. Minimum sensor node proximity, or the minimum distance between two sensor nodes, is to be $20 \mathrm{~m}$. This is because sensors placed too close to each other do not produce valuable data. Also, because the communicable proximity is $10 \mathrm{~m}$, this ensures that a single mobile relay node does not come in range of multiple sensor nodes at the same time. Also, the simulation disregards PAN connection overheads normally produced on startup and termination.

\subsection{Sensor Node Placement}

To best collect data from the environment, it is obvious that the more sensor nodes placed the better. However, it is too costly to place all desired sensor nodes in a large scale urban sensing setup. Therefore, we employed the following rules in determining the placement of sensor nodes.

\section{- Step 1: Sampling}

The target area is $5 \mathrm{~km}$ wide by $5 \mathrm{~km}$ long in which there are innumerable candidates of sensor node placement. We reduce this area to a grid 1000 units wide by 1000 units long, where each unit is $5 \mathrm{~m}$ by $5 \mathrm{~m}$. We will hereon refer to this grid as the "local map".

\section{- Step 2: Mapping and counting}

We load the pedestrian trip data along with their location data. We map the latitude and longitude on to the local map. The local map holds the number of mappings given.

Table 1. Simulation parameters.

\begin{tabular}{cc}
\hline Parameter & Value \\
\hline Center of area (lat.) & 35.689185 \\
Center of area (long.) & 139.691648 \\
Height of area & $5 \mathrm{~km}$ \\
Width of area & $5 \mathrm{~km}$ \\
Data generation rate & $1 \mathrm{~min}$ \\
Data generation sizes & $100,300,500$, \\
Communicable proximity & $700,900 \mathrm{Kbytes}$ \\
between sensor and mobile relay node & $10 \mathrm{~m}$ \\
Data transmission rate & $1600 \mathrm{kbps}$ \\
between sensor and mobile relay node & $20 \mathrm{~m}$ \\
Minimum sensor-to-sensor distance & \\
\hline
\end{tabular}


- Step 3: Sorting in ascending order by maximum latency

We place sensor nodes where the mapping count is 1 or larger and run the simulation. We measure the maximum latency (the largest value from the latencies that the particular sensor yielded throughout the simulation) against each sensor node, and once the simulation is done, we sort the sensors in ascending order by the maximum latency.

- Step 4: Deletion of sensor nodes based on node-tonode distance

Finally, we delete the sensor nodes that violate the minimum sensor node proximity. Nodes sorted to a lower rank in Step 3 are deleted first.

\subsection{Simulation Results}

Figure 4 shows the trip routes of the mobile relay nodes. Figure 5 shows the local map produced in Step 2 of Section 5.3. Points where the mapping count is 1 or more are plotted in Figure 5, amounting to 48,203 points. Figure 6 shows the sensor nodes placed in Step 4 of Section 5.3, amounting to 12,116 sensor nodes.

Figure 7 shows the maximum latencies plotted by amount of data generated. Figure 8 shows the change in uncollected data amounts and number of trips plotted by time (with a data generation amount of 300 KBytes). Uncollected data means the total amount of data stocked in all of the sensor nodes' buffers at a certain point in time. The total data size collected by the mobile relay nodes amounted to 2385 GBytes. Figure 9 sets 18,342, or the number of unique persons passing through the area during the simulation, as $100 \%$ and shows the change in maximum latency as we reduced the number of mobile relay nodes (with a data generation amount of 300 Kbytes).

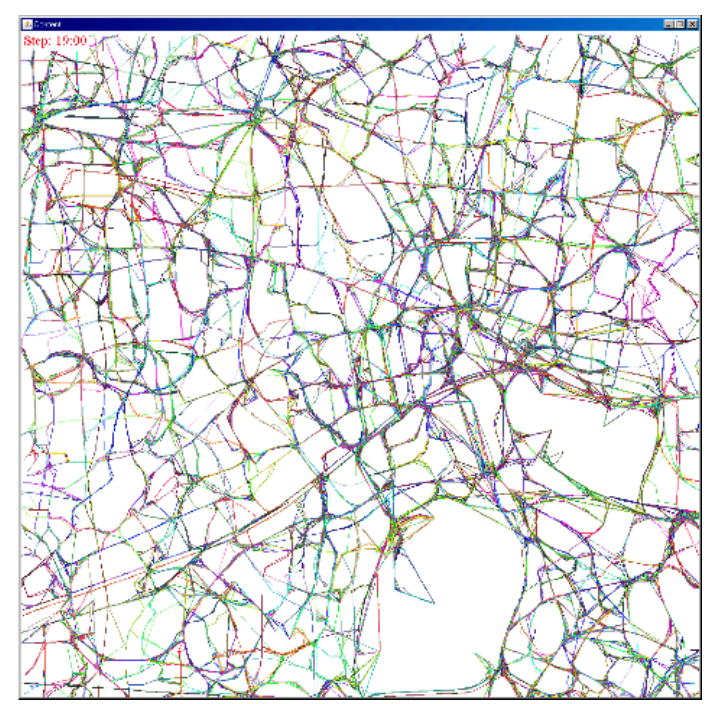

Figure 4. Trip routes of mobile relay nodes.

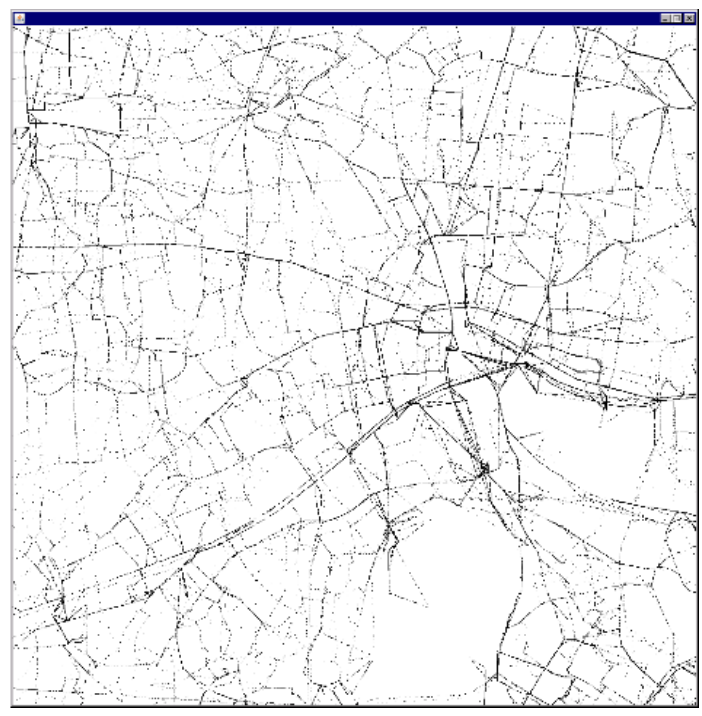

Figure 5. Mapping on the local map.

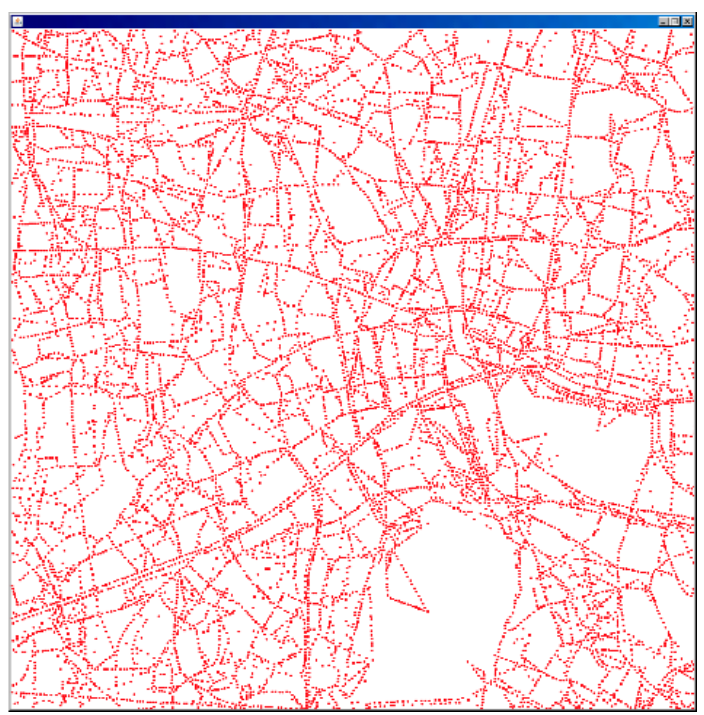

Figure 6. Sensor node placement with minimum sensor-tosensor distance in consideration.

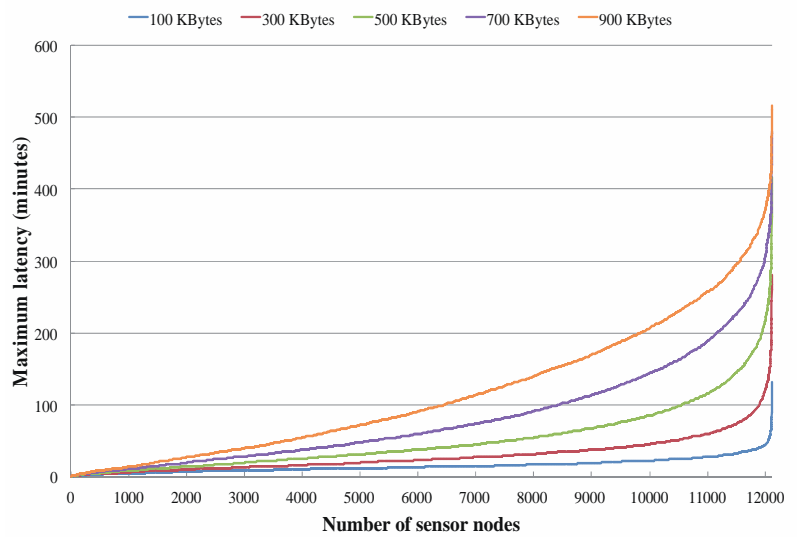

Figure 7. Change in maximum latency by amount of date generated. 


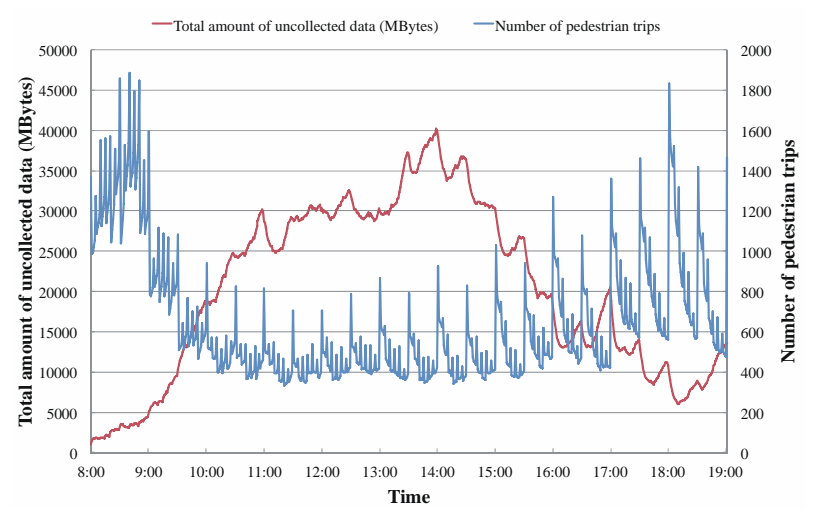

Figure 8. Changes in total amount of uncollected data and number of pedestrian trips.

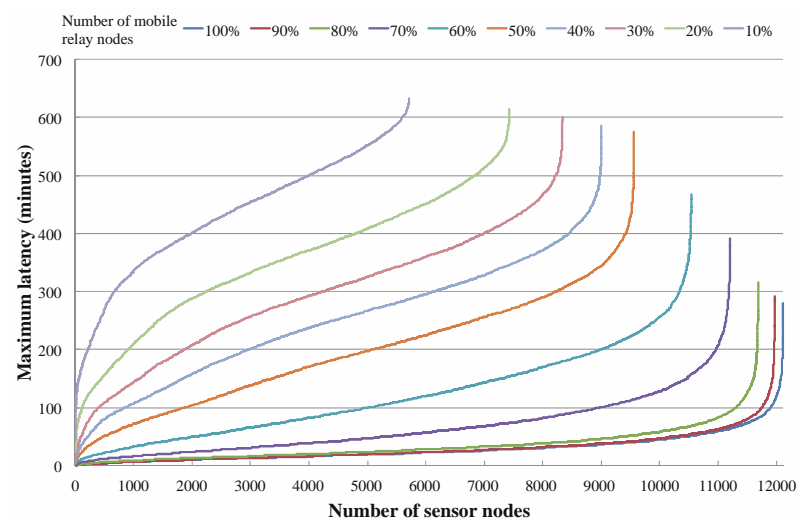

Figure 9. Change in maximum latency by number of mobile relay nodes.

\subsection{Discussion}

We reduced the actual map to a grid and used mapping counts to determine sensor node placement. This proved to be effective in recognizing the places where more people pass through, such as major roads and intersections, as shown in Figure 5. Figure 6 tells us that sensor nodes are placed with adequate distance from each other, and that sensor nodes are effectively placed in parks or shrines where motor vehicles cannot enter. Figure 7 tells us that the maximum latency increases as we increase the amount of generated data.

Let us assess these results in regard to the video surveillance system discussed in Section 3.4. Assuming that a common surveillance camera uses a $640 \times 480 \mathrm{dpi}$ resolution, MPEG-4 encoding, and a 1 FPS frame rate, data would be generated at a rate of approximately 300 Kbyte per minute. Figure 7 reads that at a 300 KByte data generation, the maximum latency ranges from $0 \mathrm{~min}$ to $280 \mathrm{~min}$ and the average is $29 \mathrm{~min}$ when the number of sensor nodes change from 0 to 12,116. This means that using this system, the surveillance system can provide all video data from approximately $30 \mathrm{~min}$ ago and older.
Now let us discuss the amount of uncollected data and the number of trips based on Figure 8. Because the area used in the simulation is a business district, the number of trips is high at time slots when people commute to and from the office. Figure 8 also shows that at time slots when there are many pedestrians, the amount of uncollected data is kept down low by the large number of mobile relay nodes.

Finally, Figure 9 tells us that the maximum latency increases as the number of mobile relay nodes decrease. This also means that not as many sensor nodes can be placed when there are fewer mobile relay nodes.

\section{Conclusions}

In recent years, urban sensing systems that provide users with valuable information using data collected with a large number of sensors is gaining demand. However, in achieving urban sensing systems, the communication channel from the sensors to the data centers pose a problem. Existing attempts to tackle this problem, such as the fixedsensor model and the roaming-sensor model, generally limit either the types of sensors used or the distances among the sensors, so they are not suitable for urban sensing systems.

In this paper, we proposed a new sensor data collection system model, the mobile relay node model, in which nodes that transmit data are physically detached from nodes that sense environment data. We also evaluated the data collection ability of the model using a simulation imitating real-world environment using traffic survey data. We have verified that the proposed system model has sufficient data collection ability for use in urban sensing systems that are not under the real-time constraint.

\section{REFERENCES}

[1] I. Akyildiz, W. Su, W. Sankarasubramaniam and E. Cayirci, "Wireless Sensor Networks A Survey," Computer Networks Elsevier Journal, Vol. 38, No. 4, 2002, pp. 393422. doi:10.1016/S1389-1286(01)00302-4

[2] G. Pottie, "Wireless Sensor Networks," Proceedings of Information Theory Workshop, 1998, pp. 139-140.

[3] K. Fall, “A Delay-Tolerant Network Architecture for Challenged Internets," Proceedings of the 2003 conference on Applications, technologies, architectures, and protocols for computer communications, 2003.

[4] K. Mohri, T. Hasegawa and R. Kurazume, "Cooperative Tracking of Pedestrian by an On-Board LRF of Mobile Robot and a Fixed Vision,” Proceedings of the 2008 JSME Conference on Robotics and Mechatronics, Vol. 2P1-H10, 2008.

[5] T. Nanri and N. Otsu, "Anomaly Detection in Motion Images Containing Multiple Persons," Transactions of Information Processing Society of Japan, Vol. 46, No. 
SIG-15, 2005, pp. 43-50.

[6] Y. Kobayashi, Y. Sato and A. Sugimoto, "Estimation of People's Trajectory by Using Sparse Distributed Cameras," Meeting on Image Recognition and Understanding, 2006, pp. 1213-1218.

[7] T. Fukatsu and M. Hirafuji, "Long-Term Monitoring System Using Field Monitoring Servers," Proceedings of AFITA/WCCA Joint Congress on IT in Agriculture, 2004, pp. 685-691.

[8] S. Guo, M. Falaki, E. Oliver, S. Rahman, A. Seth, M. Zaharia, U. Ismail and S. Keshav, "Design and Implementation of the KioskNet System," International Conference on Information Technologies and Development, 2007.
[9] A. Pentland, R. Fletcher and A. Hasson, "DakNet: Rethinking Connectivity in Developing Nations," Computer, Vol. 37, No. 1, 2004, pp. 78-83. doi:10.1109/MC.2004.1260729

[10] K. Oshima, Y. Ishida, S. Konomi and Y. Tobe, "Exploring the Uses of Pressure Sensors and Accelerometers," Proceedings of Forum of Information Technology, 2009, pp. 251-252.

[11] M. Izumi, R. Wakikawa, Y. Kawakita and Y. Akiyama, "Internet ITS Project Overview," Transactions of Information Processing Society of Japan, Vol. 43, No. 4, 2002.

[12] N. Tokitsu and K. Takahashi, "Internet ITS Project Verification Test," Transactions of Information Processing Society of Japan, Vol. 43, No. 4, 2002. 